\title{
S47. Child psychiatry
}

M.C.D.D. IN CHILDHOOD: A PRECURSER OF SCHIZOPHRENIC SPECTRUM DISORDERS

$\mathrm{H} v$ Engeland, RJ v d Gaag

Department of Child and Adolescent Psychiatry, Utrecht University, Urrecht (Netherlands)

Multiple complex developmental disorder (M.C.D.D.) is the diagnostic term for schoolage children suffering from deficits in affect modulation, high levels of anxiety, odd or disturbed relationships, poor social skills and frequent periods of mild to severe thought disorder. (COHEN et al 1986). No single DSM-III-R diagnosis provides an adequate description for this distinctive syndrome and prospective follow-up studies suggest that in adulthood many of them suffer from a schizophrenic spectrum disorder $(14-70 \%)$

We conducred rwo follow-up studies of M.C.D.D. children who were treated at our inpatient ward during the periods 1974-1980 $(n-12)$ and $1984-1990(n-43)$; mean age at follow-up 25 years and 15,4 years respectively. Raters used structured interviews (PSE, K-SADS and SCID) and were blind to the originale diagnosis. Summarizing the results, $22 \%$ of the adolescents and $64 \%$ of young adults suffered from schizophrenia spectrum disorders (2 adults were schizophrenic, the others schispectrum disorders ( 2 adults were schizophrenic, the others schi-
zoid/schizotyped personality disorders). M.C.D.D. in Childhood is a precursor for schizophrenic spectrum disorders and deserves future intensive study.

COHEN et al. (1986). J. Am. Acad. Child Adolesc. Psychiatry 25-(2) 213.220

\section{SPECIFIC AND UNSPECIFIC PREDICTORS OF UNFAVOURABLE OUTCOME IN CHILD PSYCHIATRIC DISORDER}

H Remschmidt. M.D.,Ph.D

Dept. Child \& Adolescent Psychiatry, Philipps-University, HansSachs-Str. 6, D-35033 Marburg, Germany

Prediction of outcome of psychopathological disorders is always a challenge for clinical and epidemiological research. Predictors of outcome can be subdivided into specific vs. unspecific and individual vs. psychosocial ones. Specific predictors are able to predict the quality of outcome of the special psychological disorder. In terms of more unspecific.predictors, the general level of psychopathology and not the outcome of a specific disorder can be judged. Individual predictors comprise variables as temperament, personality, and also the variability of psychopathological symptoms of a certain disorder. Psychosocial variables of predictive power are concentrated on family influences, social class, life events, and other influences outside the patients. Interactions between all of these possible predictor variables must be taken into account.

Inspite of the methodological problems of research in this field, empirical studies demonstrate that the outcome of psychopathological disorders in children and adolescents can be predicted to a high extent. This will be demonstrated by the results of empirical studies comprising conditions as eating disorders, child and adolescent schizophrenia, depression, mutism, hyperkinetic behaviour, and delinquency. 
THE COURSE OF CHILDHOOD OBSESSIVE-COMPULSIVE DISORDER P.H. Thomsen

Children's Psychiatric Hospital, Risskov (DK)

A 6 - 22 year outcome study of 47 patients with childhood onset OCD is presented.

Phenomenological aspects at baseline did not differ from that seen in children and adults in other parts of the world. Compulsive handwashing and obsessions regarding dirt and contamination were the most frequent symptoms, seen approximately in half of the patients.

At follow-up, the course of OCD was described according to 4 groups of outcome. Approximately one fourth of the patients fell in each group:

1. No OCD in adulthood;

2. Subclinical OCD symptoms in adulthood

3. An episodic course of $O C D$ in adulthood

4. Chroric OCD in adulthood.

In practically all cases where psychopathology was present, OCD was the main disorder. The intraindividual continuity of specific obsessive-compulsive symptoms was low.

Frequent comorbid symptoms at follow-up were depression and symptoms of anxiety.

Probands with OCD at follow-up had significantly lower social functioning than probands without OCD.
WHAT PREDICTS THE COURSE OF SCHIZOAFFECTIVE DISORDER STARTING IN ADOLESCENCE

MH Schmidt

Central Institute of Mental Health, Department of Child Psychiatry, J5

D-68159 Mannheim (Germany)

Within a follow up sample of 118 patients (64 males, 54 females) firs hospitalized before age 18 for any kind of schizophrenic disorder, we found 13 with schizoaffective episodes. In average they were 23 years old at reassesment, average catamnestic interval was 7 years. We found remarkable comorbidity with mental retardation.

For the analysis number of schizoaffective episodes and combination with schizophrenic and affective episodes was taken into account. Social behaviour a discharge after first admission was found to be the best predictor for later outcome measured by school/work performance and social disability. Outcome was less favourable than described for adults with schizoaffective disorder and more related to that of schizophrenic disorder starting in early age than to the course of affective disorder.

\section{CONTINUITY AND CHANGE OF PSYCHOPATHOLOGY FROM CHILDHOOD INTO ADULTHOOD: A LONGITUDINAL GENERAL POPULATION STUDY FC Verhulst. RF Ferdinand \\ Department of Child and Adolescent Psychiatry, Sophia Children's Hospital/Erasmus University Rotterdam, Dr. Molewaterplein 60, 3015 GJ Rotterdam, The Netherlands.}

A general population sample of 2,076 4-to-16 year old Dutch children and adolescents has been followed-up across an 8-year time span.

At 2-year intervals psychopathology was assessed using standardized procedures. This study focusses on the prediction of psychopathology from (early) adolescence into young adulthood. From the original sample of 92511 . to-16-year olds at Time 1 , we received usable information on 706 subjects aged 19- to 24-years at follow-up (76.3\%).

Subjects completed the Young Adult Self-Report (YASR), a self-report measure for the assessment of a wide range of psychopathology. Based on the YASR scores, a subsample of 131 subjects was selected and subjected to standardized clinical interviews revealing DSM diagnoses and judgements of global functioning.

The results of this study in terms of prediction of psychopathology across a rather long time interval covering adoiescence and young adulthood will be presented. 